\title{
Recruitment of mesenchymal stem cells by stromal cell-derived factor $1 \alpha$ in pulp cells from deciduous teeth
}

\author{
YUKI AKAZAWA $^{1}$, TOMOKAZU HASEGAWA ${ }^{2}$, YOSHITAKA YOSHIMURA ${ }^{3}$, NAOYUKI CHOSA $^{4}$, \\ TAKEYOSHI ASAKAWA ${ }^{5}$, KIMIKO UEDA ${ }^{1}$, ASUNA SUGIMOTO ${ }^{2}$, TAKAMASA KITAMURA ${ }^{2}$, \\ HIROSHI NAKAGAWA ${ }^{1}$, AKIRA ISHISAKI ${ }^{4}$ and TSUTOMU IWAMOTO ${ }^{1,2}$
}

${ }^{1}$ Department of Pediatric Dentistry, Tokushima University Hospital; ${ }^{2}$ Department of Pediatric Dentistry, Institute of Health Biosciences, University of Tokushima Graduate School, Tokushima 770-8504;

${ }^{3}$ Department of Molecular Cell Pharmacology, Division of Oral Pathological Science, Hokkaido University

Graduate School of Dental Medicine, Sapporo, Hokkaido 060-8586; ${ }^{4}$ Department of Oral Biochemistry,

School of Dentistry, Iwate Medical University, Morioka, Iwate 020-8505; ${ }^{5}$ Department of Special Needs Dentistry, Division of Dentistry for Persons with Disabilities, Showa University School of Dentistry, Tokyo 145-8515, Japan

Received February 4, 2015; Accepted May 19, 2015

DOI: $10.3892 /$ ijmm.2015.2247

\begin{abstract}
Dental pulp cells (DPCs), including dental pulp (DP) stem cells, play a role in dentine repair under certain conditions caused by bacterial infections associated with caries, tooth fracture and injury. Mesenchymal stem cells (MSCs) have also been shown to be involved in this process of repair. However, the mechanisms through which MSCs are recruited to the DP have not yet been elucidated. Therefore, the aim of the present in vitro study was to investigate whether stromal cell-derived factor $1 \alpha$ (SDF1)-C-X-C chemokine receptor type 4 (CXCR4) signaling is involved in tissue repair in the DP of deciduous teeth. A single-cell clone from DPCs (SDP11) and UE7T-13 cells were used as pulp cells and MSCs, respectively. The MG-63 and HuO9 cells, two osteosarcoma cell lines, were used as positive control cells. Reverse transcription polymerase chain reaction (RT-PCR) revealed that all cell lines (SDP11, UE7T-13 MG-63 and HuO9) were positive for both $S D F 1$ and $C X C R 4$ mRNA expression. Moreover, immunocytochemical analysis indicated that SDF1 and CXCR4 proteins were expressed in the SDP11 and UE7T-13 cells. SDF1 was also detected in the cell lysates (CLs) and conditioned medium (CM) collected from the SDP11 and UE7T-13 cells, and AMD3100, a specific antagonist of CXCR4, inhibited the migration of the UE7T-13 cells; this migration was induced by treatment with CM, which was collected from the SDP11 cells. In addition, real-time PCR showed that the expression of SDF1 in the SDP11 cells was inhibited by treatment with
\end{abstract}

Correspondence to: Dr Tomokazu Hasegawa, Department of Pediatric Dentistry, Institute of Health Biosciences, University of Tokushima Graduate School, 3-18-15 Kuramoto, Tokushima 770-8504, Japan

E-mail: hasegawa@tokushima-u.ac.jp

Key words: stromal cell-derived factor $1 \alpha, \mathrm{C}-\mathrm{X}-\mathrm{C}$ chemokine receptor type 4, pulp cells, deciduous teeth
$20 \mathrm{ng} / \mathrm{ml}$ fibroblast growth factor (FGF)-2, and exposure to AZD4547, an inhibitor of the FGF receptor, blocked this inhibition. Collectively, these data suggest that SDF1 produced by DP plays an important role in homeostasis, repair and regeneration via the recruitment of MSCs.

\section{Introduction}

The hard tissue of teeth consists of enamel and dentin. Enamel, which covers the crown, is formed by ameloblasts derived from the dental epithelium, which never regenerates after tooth eruption. Dentin, which is a major component of teeth, is formed by odontoblasts derived from neural crest cells and includes connective tissue, termed dental pulp (DP), in the center. The DP is a non-hematopoietic connective tissue that is almost completely surrounded by dentin $(1,2)$. Odontoblasts are located peripherally on the inside wall of the DP chamber, and they secrete dentin. After tooth maturation, the DP is protected only by reparative or secondary dentin, which is formed by odontoblasts in response to general mechanical stress or disruption and dentinal degradation caused by bacterial infections. In addition, dental pulp progenitor/stem cells (DPSCs) exist in dental pulp and become activated in response to odontoblast injury. DPSCs are multipotent stem cells that are capable of differentiating into odontoblasts, osteoblasts, adipocytes and neural cells in vivo (3). DPSCs are thought to be useful for dentin regeneration. However, there are very few DPSCs in pulp tissue (4). Mesenchymal stem cells (MSCs) are also multipotent stem cells that can differentiate not only into osteoblastic and chondroblastic cell lineages, but also into adipocytes, tendon and muscle $(3,5)$. It has been demonstrated that MSCs can differentiate into dentin-forming odontoblasts and participate in dentin regeneration (3). However, the mechanisms through which MSCs migrate into the DP have not yet been elucidated.

Stromal cell-derived factor $1 \alpha$ (SDF1, also known as CXCL12) is an $\alpha$-chemokine that attracts MSCs and endothelial progenitor cells (EPCs) through interaction with its unique 
receptor, C-X-C chemokine receptor type 4 (CXCR4) (6-9). In adults, tissue repair and regeneration, complex processes involving the proliferation and migration of tissue stem celldependent progenitor cells, are thought to involve the selective recruitment of circulating or resident stem cell populations. The expression of SDF1 in injured tissue correlates with the recruitment of adult stem cells and tissue regeneration, highlighting the importance of SDF1 in stem and progenitor cell recruitment (6-9). Thus, SDF1 may play an important role in coordinating tissue injury and repair in DP. However, the exact which SDF1 plays in relation to dental pulp cells (DPCs) derived from human teeth is not known.

Therefore, in the present study, we sought to determine the functions of SDF1 and CXCR4 in DPC cultures and human MSCs. To the best of our knowledge, this is the first report describing the expression of SDF1 and CXCR4 in DPCs derived from human deciduous teeth.

\section{Materials and methods}

Cell culture. The UE7T-13 cells, a human bone marrow-derived MSC line infected with retroviruses expressing papillomavirus E7 and hTERT in order to prolong the life span of the cells (10-13), were purchased from the Health Science Research Resources Bank (JCRB1154; Japan Health Sciences Foundation, Tokyo, Japan) and cultured in $\alpha$-modified minimum essential medium ( $\alpha$-MEM; Gibco-BRL, Gaithersburg, MD, USA) supplemented with $10 \%$ fetal bovine serum (FBS; Gibco-BRL). The MG-63 cells derived from human osteosarcoma (14) were purchased from the American Type Culture Collection (CRL-1427; ATCC, Manassas, VA, USA). The HuO9 human osteosarcoma cells (15) were purchased from the Japan Health Sciences Foundation (JCRB0427) and cultured in $\alpha$-MEM containing $10 \% \mathrm{FBS}$. All cultures were maintained at $37^{\circ} \mathrm{C}$ in a humidified atmosphere of $5 \% \mathrm{CO}_{2}$.

Isolation of DPCs. The DP tissues were obtained from the crown and root of healthy human deciduous teeth (obtained from 3 donors, aged 6-8 years). Informed consent was obtained from the donors' parents prior to tooth extraction, which was carried out at Tokushima University Hospital (Tokushima, Tokyo) during the course of orthodontic treatment. The study protocol was approved by the Ethics Committee of Iwate Medical University, School of Dentistry, Morkioka Japan (no. 01101) and Tokushima University Hospital, Tokushima, Japan.

The DP tissues were cut into sections using a surgical blade and were digested with collagenase $(2 \mathrm{mg} / \mathrm{ml})$ at $37^{\circ} \mathrm{C}$ for $30 \mathrm{~min}$. The tissues were then washed with Dulbecco's phosphate-buffered saline (DPBS), placed in culture dishes and maintained in $\alpha$-MEM supplemented with $10 \%$ FBS. Fibroblastic cells that grew from the DP tissues were used as DPCs. When the cells reached confluence, they were detached with $0.2 \%$ trypsin and $0.02 \%$ ethylenediaminetetraacetic acid tetrasodium salt (EDTA-4Na) in DPBS (Gibco-BRL) and subcultured at a split ratio of 1:4, as previously described (1).

Transfection of DPCs with the hTERT gene. The DPCs were transfected with the $p B A B E-n e o-h T E R T$ plasmid, which harbored a neomycin-resistance gene (Addgene Inc., Cambridge, MA, USA) using Lipofectamine LTX (Invitrogen
Life Technologies, Carlsbad, CA, USA) according to the manufacturer's instructions. The cells were exposed to $\alpha$-MEM containing 10\% FBS and $200 \mu \mathrm{g} / \mathrm{ml} \mathrm{G} 418$ (Gibco-BRL) for 12-15 days. The surviving cells were trypsinized and cultured further in 100-mm culture dishes, as previously described (16).

Single-cell cloning. Single-cell clones were obtained using the limited dilution method as previously described (16). Clones obtained after single-cell cloning were named single cell clones derived from human deciduous tooth pulp cells (termed SDPCs). All the experiments were performed using clone no. 11 (SDP11 cells) cultured in $\alpha$-MEM supplemented with $10 \%$ FBS in the absence or presence $10 \mathrm{ng} / \mathrm{ml}$ fibroblast growth factor (FGF)-2 (R\&D Systems, Minneapolis, MN, USA) for 2 days. All cultures were maintained at $37^{\circ} \mathrm{C}$ in a humidified atmosphere of $5 \% \mathrm{CO}_{2}$ in air.

Isolation of total RNA. Total RNA was extracted from the cultured cells using TRIzol reagent (Invitrogen Life Technologies) as previously described (16). The total RNA pellet was washed briefly with $75 \%$ ethanol, resuspended in $30 \mu 1$ diethylpyrocarbonate (DEPC)-treated water (Invitrogen Life Technologies) and stored at $-80^{\circ} \mathrm{C}$. The concentration of total RNA was determined spectrophotometrically by measuring the optical density at $260 \mathrm{~nm}$, using a BioPhotometer (Eppendorf, Hamburg, Germany).

Reverse transcription polymerase chain reaction (RT-PCR). RNA $(1 \mu \mathrm{g})$ was reverse transcribed into first-strand cDNA using a PrimeScript RT reagent kit (Takara Bio, Kyoto, Japan), according to the manufacturer's instructions. cDNA samples were then amplified with specific primer pairs (listed in Table I) and as previously described: for SDF1 (30 cycles) (16), CXCR4 (35 cycles) (17) and glyceraldehyde 3-phosphate dehydrogenase (GAPDH, 28 cycles) (16). PCR was performed for the given number of cycles at $94^{\circ} \mathrm{C}$ for $1 \mathrm{~min}, 56^{\circ} \mathrm{C}$ for $1 \mathrm{~min}$ and $72^{\circ} \mathrm{C}$ for $1 \mathrm{~min}$. Subsequently, $8 \mu 1$ of the PCR product were resolved on a $4.0 \%$ agarose gel and stained with ethidium bromide. After staining, the bands were visualized using an imaging system (AE-6932GXCF; ATTO, Tokyo, Japan).

Real-time PCR. One microgram of the RNA sample was reverse transcribed into first-strand cDNA using a PrimeScript RT reagent kit (Takara Shuzo Co., Ltd., Kyoto, Japan), according to the manufacturer's instructions and as previously described $(1,16)$. A Thermal Cycler Dice real-time system (Takara Shuzo Co., Ltd.) was used for the two-step RT-PCR. The cDNA was amplified with SYBR Premix Ex Taq and specific oligonucleotide primers for the target sequences encoding parts of SDF1 and CXCR4. The primers (listed in Table I and as previously described) were designed based on the cDNA sequences of human mRNA for SDF1 (16), CXCR4 (17) and GAPDH (16). The amplification conditions were as follows: $10 \mathrm{sec}$ at $95^{\circ} \mathrm{C}$, followed by 40 cycles of $95^{\circ} \mathrm{C}$ for $5 \mathrm{sec}$ and $60^{\circ} \mathrm{C}$ for $30 \mathrm{sec}$, with a final $15 \mathrm{sec}$ at $95^{\circ} \mathrm{C}$ and $30 \mathrm{sec}$ at $60^{\circ} \mathrm{C}$ in the Thermal Cycler Dice real-time system.

Western blot analysis of SDF1 protein expression in SDP11 cells. The cells were plated at a density of $1.5 \times 10^{5}$ cells/dish (60 $\mathrm{mm}$ in diameter) and cultured for 2 days. The cells were 
Table I. Primers used for PCR in the present study.

\begin{tabular}{lllc}
\hline Gene name & Primer & \multicolumn{1}{c}{ Oligonucleotide sequence $\left(5^{\prime} \rightarrow 3^{\prime}\right)$} & Predicted size $(\mathrm{bp})$ \\
\hline SDF1 & Forward & GAGCCAACGTCAAGCATCTCAA & 109 \\
& Reverse & TTTAGCTTCGGGTCAATGCACA & \\
FXCR4 & Forward & AGCTGTTGGCTGAAAAGGTGGTCTATG & 260 \\
& Reverse & GCGTTCTGGTGGCCCTTGGAGTGTG & 267 \\
\hline
\end{tabular}

SDF1, stromal cell-derived factor $1 \alpha$; CXCR4, C-X-C chemokine receptor type 4.

then washed twice with DPBS and treated with lysis buffer (10 mM HEPES-KOH (pH 7.5), $100 \mathrm{mM} \mathrm{KCl}$ and 0.1\% NP-40]. Conditioned medium (CM) was also collected. The protein concentrations in the cell lysates (CLs) and CM were measured using a Bio-Rad protein assay kit (Bio-Rad, Hercules, CA, USA). Equal amounts of protein $(20 \mu \mathrm{g})$ from each sample were separated by sodium dodecyl sulfate-polyacrylamide gel electrophoresis (SDS-PAGE) on 10\% gels and then transferred onto polyvinylidene difluoride membranes (Millipore, Bedford, MA, USA). After blocking with 5\% skim milk in Tris-buffered saline containing $0.1 \%$ Tween-20 (TBST), the membranes were incubated with rabbit anti-human SDF1 antibody (Cat. no. 500-P87A; PeproTech, Rocky Hill, NJ, USA) and subsequently with anti-mouse secondary antibody (product no. 31432; Zymed Laboratories Inc., San Francisco, CA, USA). Specific protein bands on the membrane were detected using an enhanced horseradish peroxidase (HRP) conjugate substrate kit (Bio-Rad) as previously described (1).

Immunocytochemistry. The cells were fixed with cold 4\% paraformaldehyde in $0.1 \mathrm{M} \mathrm{DPBS}$ (4) for $15 \mathrm{~min}$. The cells were then rinsed in $1 \mathrm{X}$ Tris-buffered saline (TBS), blocked with $10 \%$ normal goat serum(NGS) in $1 \mathrm{X}$ Triton X-100 in TBS for $1 \mathrm{~h}$, and incubated with primary antibodies overnight. The following primary antibodies were used: antibodies against SDF1 (1:1,000; PeproTech) and CXCR4 (1:1,000; Cat. no. MAB172-SP; R\&D Systems). After washing in TBS, the cells were incubated for $1 \mathrm{~h}$ at room temperature with the appropriate secondary antibodies [anti-mouse Alexa 594 (Cat. no. R37115) at a dilution of 1:1,000 or anti-rabbit Alexa 488 (Cat. no. A-11034) at a dilution of 1:1,000 (both from Invitrogen Life Technologies)]. Finally, the nuclei were stained with 4',6-diamidino-2-phenylindole (DAPI). The cells were then mounted on coverslips in ProLong anti-fade reagent (Invitrogen Life Technologies) and examined under an epifluorescence microscope (Nikon ECLIPSE TE2000-U; Nikon Corp., Tokyo, Japan).

Transmembrane migration assay. Cell migration was evaluated using a Transwell system as previously described (10). Chambers with a $8-\mu \mathrm{m}$ pore size and $6.4 \mathrm{~mm}$ in diameter were used (Corning Cell Culture Insert 24-well System; Sigma-Aldrich, St. Louis, MO, USA). The UE7T-13 cells were treated with DPBS or $0.1 \mu \mathrm{M}$ AMD3100 (a specific antagonist of CXCR4; Millipore, Darmstadt, Germany) for $3 \mathrm{~h}$ prior to the assay. The cells were then dissociated and re-seeded into

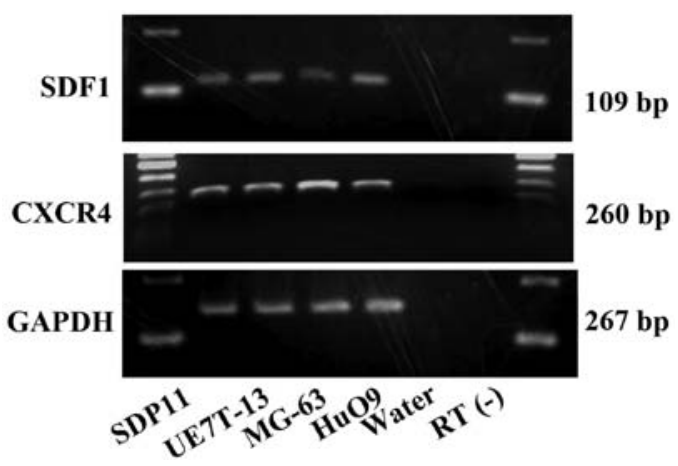

Figure 1. RT-PCR analysis of stromal cell-derived factor $1 \alpha$ (SDF1) and C-X-C chemokine receptor type 4 (CXCR4) in SDP11, UE7T-13, MG-63 and HuO9 cells. SDP11, UE7T-13, MG-63 and HuO9 cells were used as positive controls. Water was used in the place of the RT product. RT (-), total RNA from SDP11 cells was used in place of the RT product.

the upper chamber at a density of $1 \times 10^{5}$ cells $/ \mathrm{ml}$. Subsequently, $700 \mu \mathrm{l}$ of CM collected from the SDP11 cell culture were added to the lower well. Following a 6 -h incubation at $37^{\circ} \mathrm{C}$, the cells migrating through the membrane were fixed in $4 \%$ paraformaldehyde for $15 \mathrm{~min}$, while the cells on the upper surface of the inserts were removed using cotton-tipped swabs. The Transwell chamber was immersed in $1 \mathrm{~g} / \mathrm{ml}$ hematoxylin (Sigma-Aldrich) for $15 \mathrm{~min}$. To quantify the migrating cells, 5 random microscopic fields per membrane were photographed using a Nikon inverted microscope system at x40 magnification. All assays were performed independently 3 times.

Effect of FGF-2 on SDF1 expression. In order to determine whether $S D F 1$ expression can be modulated by FGF-2 (Cat. no. 233-FB-01M; R\&D Systems), the SDP11 cells were cultured in the presence or absence of $20 \mathrm{ng} / \mathrm{ml} \mathrm{FGF-2}$ for 2 days. To confirm whether FGF-2 acts through the FGF receptor (FGFR), $1 \mu \mathrm{M}$ AZD4547 (Cat. no. sc-364421, Santa Cruz Biotechnology, Inc., Dallas, TX, USA) was also used as a specific inhibitor of FGFR. Following culture, RNA was extracted and real-time PCR was performed as described above.

Statistical analysis. The results are expressed as the means \pm SEM. Statistical significance was determined using one-way analysis of variance and the Bonferri correction method between pairs of groups. Differences with P-values of $<0.01$ were considered statistically significant. 

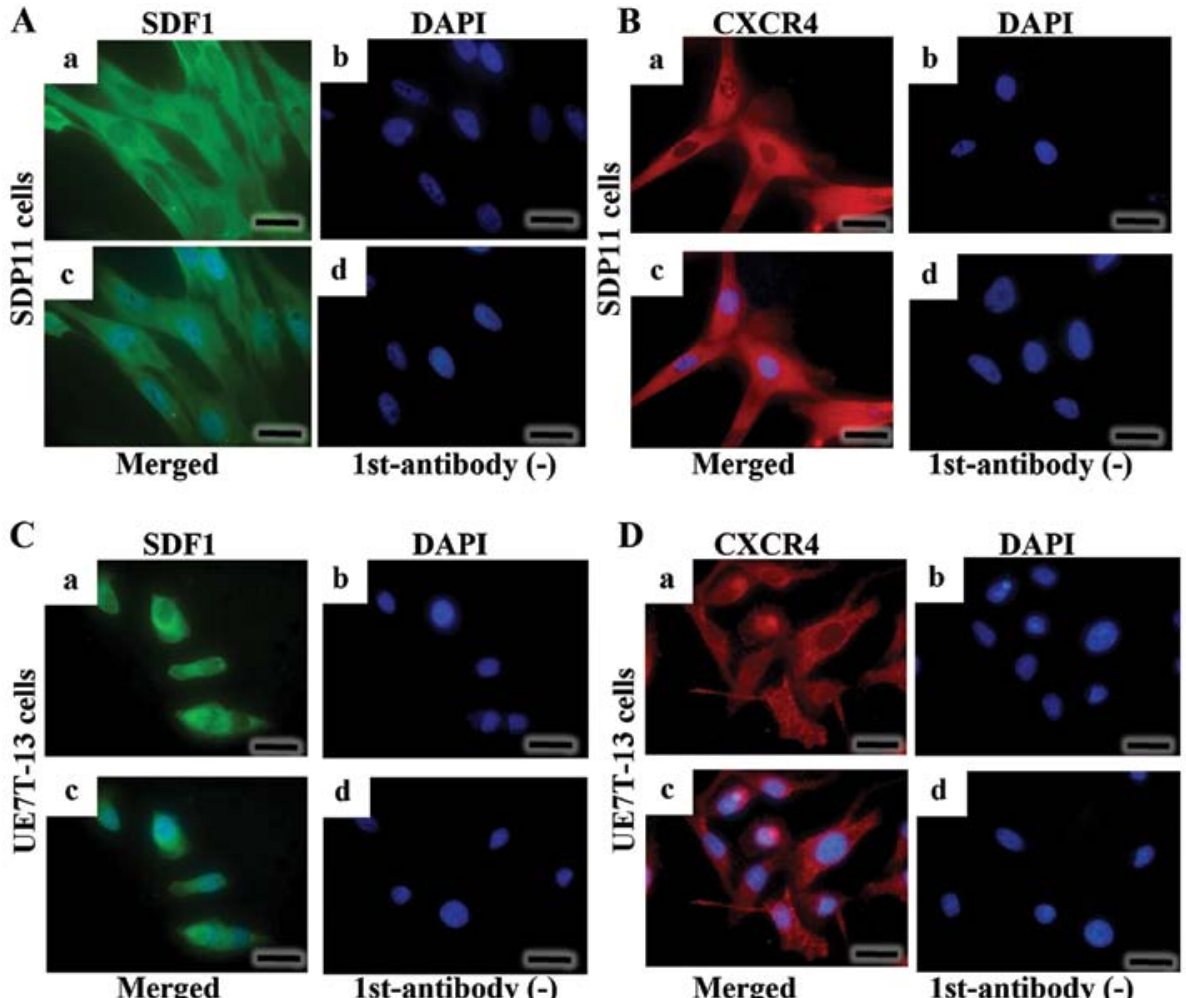

Merged

1st-antibody (-)

Figure 2. Immunocytochemical analysis of stromal cell-derived factor $1 \alpha$ (SDF1) and C-X-C chemokine receptor type 4 (CXCR4) in SDP11 and UE7T-13 cells. (A and B) Staining of SDP11 cells with anti-SDF1 and anti-CXCR4 antibodies, respectively. (A-a) SDF1 expression in SDP11 cells (green); (A-b) immunofluorescence imaging of nuclei stained with DAPI (blue); (A-c) merged images of (a and b); (A-d) omission of the primary antibody for SDF1. (B-a) CXCR4 expression in SDP11 cells (red); (B-b) staining of nuclei using DAPI (blue); (B-c) merged image of (a and b); (B-d) omission of the primary antibody for CXCR4. (C and D) Staining of UE7T-13 cells with anti-SDF1 and anti-CXCR4 antibodies, respectively. (C-a) SDF1 expression in UE7T-13 cells (green); (C-b) staining of nuclei with DAPI (blue); (C-c) merged images of (a and b); (C-d) omission of the primary antibody for SDF1; (D-a) CXCR4 in UE7T-13 cells (red); (D-b) immunofluorescence imaging of nuclei stained with DAPI (blue); (D-c) merged images of (a and b); (D d) omission of the primary antibody for CXCR4. Scale bars, $50 \mu \mathrm{m}$.

A

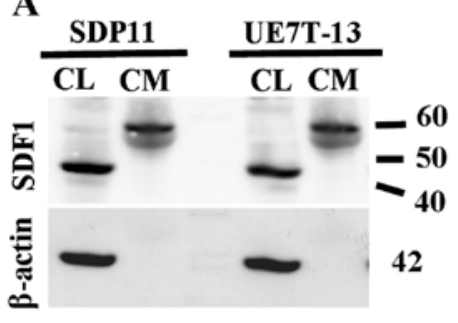

B

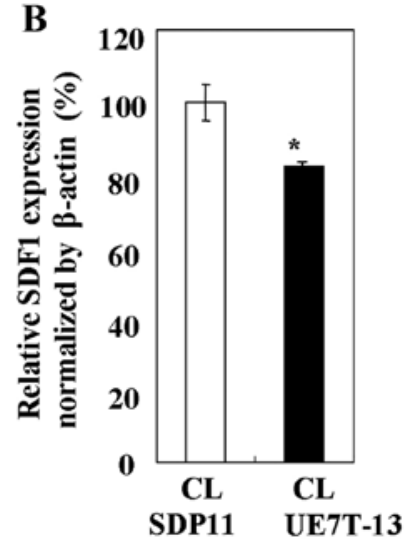

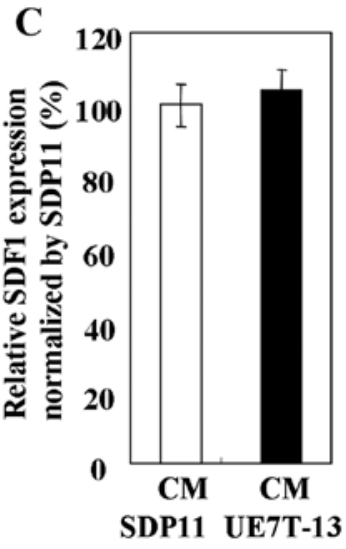

Figure 3. Western blot analysis of stromal cell-derived factor $1 \alpha$ (SDF1) in SDP11 and UE7T-13 cells. (A) SDF1 expression in cell lysates (CLs) and conditioned medium (CM) collected from SDP11 and UE7T-13 cells. Expression of $\beta$-actin was also monitored as a loading control. (B) Densitometric analysis of SDF1 expression in CLs following normalizatino to $\beta$-actin expression. SDF1 expression in the CLs of SDP11 cells was set as $100 \%$. (C) Densitometric analysis of SDF1 expression in CM. SDF1 expression in SDP11 cells was set as $100 \%$, and data are presented as fold increases. Data represent the means \pm SEM. ${ }^{*} \mathrm{P}<0.05$.

\section{Results}

Expression of SDF1 and CXCR4 in SDP11 cells. We first confirmed the mRNA expression of SDF1 and CXCR4 in the SDP11, MG-63, HuO9 and UE7T-13 cells using RT-PCR (Fig. 1). SDF1 and CXCR4 mRNA was observed in all the cell lines examined in the present study (Fig. 1). No bands were obserbed for the water and RT(-) negative controls.
Immunocytochemical analysis of SDF1 and CXCR4. The SDP11 and UE7T-13 cells were stained using anti-SDF1 antibody (Fig. 2A-a and C-a). SDF1 presented as diffuse cytoplasmic fluorescent green signals, with no staining in the nuclei (Fig. 2A-a and C-a). No signals were observed when the primary antibody was omitted (Fig. 2A-d and C-d). CXCR4 was detected in both the cytoplasmic and cell surface compartments (Fig. 2B-a and D-a). No signal was 

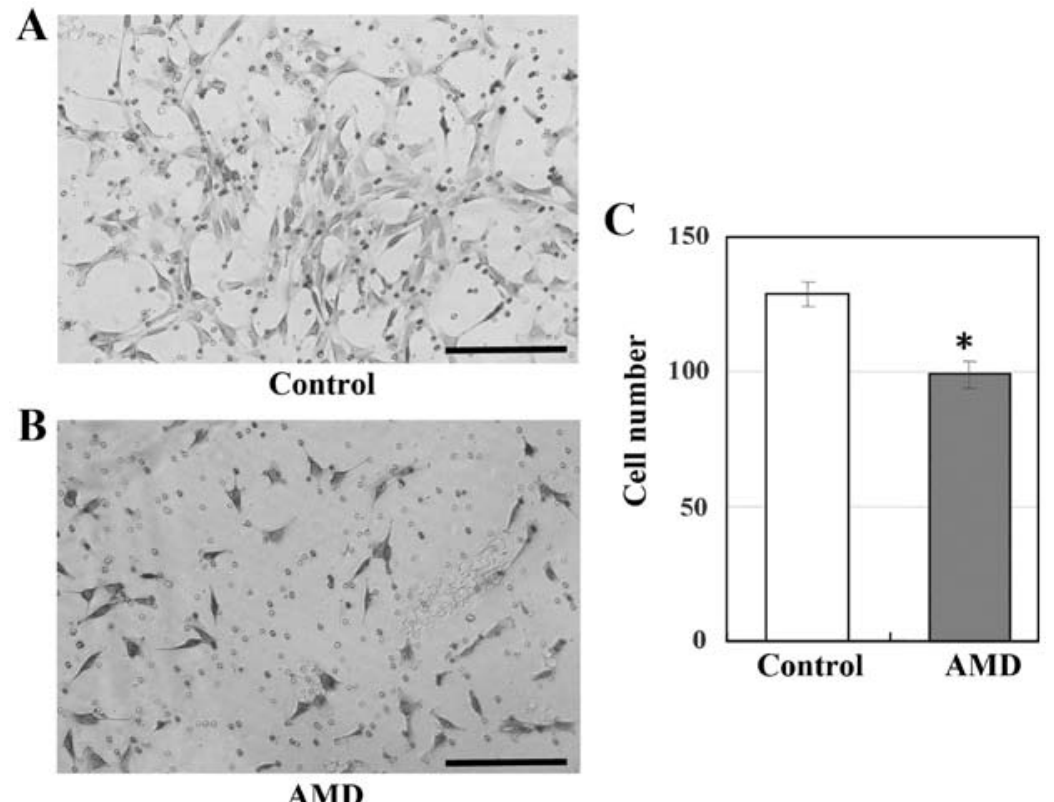

AMD

Figure 4. Effects of conditioned medium (CM) collected from SDP11 cells on chemotactic migration of UE7T-13 cells. (A) UE7T-13 cell migration following induction by CM. (B) UE7T-13 cell migration following treatment with AMD3100 (a specific antagonist of CXCR4). (C) Graph depicting UE7T-13 cell migration following treatment with AMD3100. Scale bars, $200 \mu \mathrm{m}$. Data represent the means \pm SEM. ${ }^{*} \mathrm{P}<0.05$.

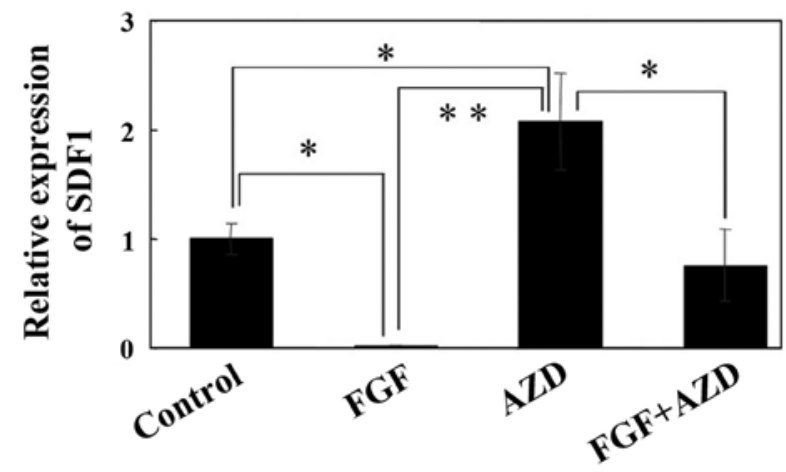

Figure 5. Stromal cell-derived factor $1 \alpha$ (SDF1) expression was inhibited by treatment with fibroblast growth factor (FGF)-2. Analysis of SDF1 expression in SDP11 cells following treatment with FGF-2 alone, AZD4547 (an inhibitor of FGF receptor) alone or combination treatment with FGF-2 and AZD4547. Data represent means \pm SEM. ${ }^{*} \mathrm{P}<0.05,{ }^{* *} \mathrm{P}<0.01$.

detected when the primary antibody for CXCR4 was omitted (Fig. 2B-d and D-d).

Western blot analysis of SDF1. To determine the protein expression of SDF1, whole CLs and CM were prepared from the SDP11 and UE7T-13 cells. The expression of SDF1 was detected in the CLs and CM from both cell lines using antiSDF1 antibody (Fig. 3). The band representing SDF1 appeared at a higher molecular weight than the theoretical molecular weight, possibly due to either an association of the chemokine with other molecules or the aggregation of the chemokine, as SDF1 is able to form oligomers spontaneously (18). The highest levels of SDF1 protein in all samples tested were observed in the CLs from the SDP11 cells (Fig. 3B). SDF1 expression was significantly higher in the CLs from the SDP11 cells than in the CLs from the UE7T-13 cells (Fig. 3B); however, no significant differences (very slight difference) in SDF1 expression were observed between the CM of the SDP11 and UE7T-13 cells (Fig. 3C).

Transmembrane migration assay. To determine whether the inhibition of CXCR4 in UE7T-13 cells inhibits cell migration, the cell cultures were treated with the CXCR4-specific inhibitor, AMD3100. As shown in Fig. 4, the migration of the UE7T-13 cells was significantly inhibited by treatment with AMD 3100 .

SDF1 expression is regulated by FGF-2. Subsquently, the effects of FGF-2 on SDF1 expression were evaluated by real-time PCR. The results of real-time PCR revealed that SDF1 expression was substantially inhibited by treatment with $20 \mathrm{ng} / \mathrm{ml} \mathrm{FGF-2} \mathrm{for} 48 \mathrm{~h}$ in the SDP11 cells (Fig. 5). By contrast, treatment with AZD4547 (an inhibitor of the FGF receptor) alone significantly increased $S D F 1$ expression, while combined treatment with FGF-2 and $1 \mu \mathrm{M}$ AZD4547 did not significantly alter $S D F 1$ expression (Fig. 5).

\section{Discussion}

In the present study, we examined the expression of the chemokine, SDF1, and its receptor, CXCR4, in human DPCs and MSCs. We found that both proteins were expressed in the CLs and CM from the SDP11 and UE7T-13 cells. Moreover, the inhibition of the CXCR4 using the specific inhibitor, AMD3100, in the UE7T-13 cells markedly inhibited cell migration. These data highlight the potential roles of SDF1 and CXCR4 in a variety of biological processes in DP.

SDF1 is a chemokine that was first characterized as a growth-stimulating factor in B cell precursors $(19,20)$. CXCR4, the physiological receptor of SDF1, is a seventransmembrane receptor coupled to heterotrimeric guanosine triphosphate(GTP)-binding proteins (21). In a previous study, the conditional knockout of CXCR4 in mice demonstrated that 
SDF1-CXCR4 signaling is essential to the maintenance of the hematopoietic stem cell pool in the bone marrow stromal cell niche (22). Furthermore, SDF1 is upregulated in response to injury from certain pathological conditions, such as diabetes, liver cirrhosis and myocardial infarction, and promotes the migration and differentiation of residential stem cells (23-26). Therefore, SDF1 plays a critical role in a variety of cellular functions, including embryogenesis, tissue homeostasis and inflammation response.

In healthy human DP, 17 chemokine genes are expressed, including SDF1, suggesting that locally produced chemokines are involved in the migration of immune cells during bacterial infections (27). Zhou et al demonstrated that the engraftment of bone marrow-derived cells (BMDCs) leads to their migration into DP tissue, indicating that BMDCs may participate in the regeneration of dentin, thereby serving as a source of stem cells which can replace odontoblasts $(28,29)$. As SDF1 expression is significantly higher in dental tissue than in other tissues (e.g., adipose, lung and liver tissue), the SDF1-CXCR4 pathway is likely involved in the engraftment of BMDCs (28). Hence, these residential stem cells are capable of homing in on inflammatory sites in response to injury signals, such as dental decay, and differentiating into odontoblasts which repair dentin. In the present study, we demonstrated DPCs from deciduous teeth (i.e., SDP11 cells) expressed SDF1 and CXCR4. Indeed, the migration of the UE7T-13 cells was observed in the presence of CM. When the UE7T-13 cells were treated with AMD3100, a CXCR4 inhibitor, the cell migration was inhibited. These results showed that the activity of SDF1 in CM was inhibited by the CXCR4 inhibitor, AMD3100. From the above results, it is demonstrated that SDF1 produced by pulp cells regulates the migration of MSCs/ DPSCs in DP.

It has been reported that healthy tissues basally express SDF1 (30). In addition, SDF1 is widely expressed in various tissues and cell lines, with the exception of blood (31). Our data are consistent with those of previous reports $(30,31)$. Moreover, SDP11 cells from DP constitutively expressed SDF1 mRNA at a level similar to that of GAPDH (data not shown). Thus, these data suggest that pulp cells attract and maintain DSPCs/MSCs in the pulp chamber under normal conditions and that they participate in the response to abnormal conditions, such as infection. SDF1 also promotes EPC migration in a concentration-dependent manner (8). Therefore, it is reasonable to speculate that DPSCs/MSCs and EPCs may be induced to migrate to damaged sites, where they can then participate in tissue repair. Thus, since there are both MSCs and EPCs in bone marrow, circulatory system and areas surrounding DP as residential stem cells, these cells may be involved in a coordinating mechanism for pulpal tissue regeneration, which operates when pulp tissues are damaged.

It has been demonstrated in previous studies that a number of cytokines and bioactive materials have clinical uses $(32,33)$. One such cytokine molecule, FGF-2, is a multifunctional growth factor that exerts various effects, including the induction of proliferation and differentiation of a wide range of mesodermal and neuroectodermal cells $(10,32,33)$. FGF-2 is also a crucial factor in wound healing and is involved in the induction of angiogenesis, cell proliferation and the accumulation and modulation of the extracellular matrix (34). Under pathological conditions, such as moderate caries, growth factors such as
FGF-2 may be released from the dentin and travel to pre-existing odontoblasts in order to secrete reparative dentin (35). Moreover, compared with erythropoietin (EPO), interleukin (IL)-6, SDF1 $\beta$ and vascular endothelial growth factor (VEGF), FGF-2 is the most potent effector of MSC migration (36). In light of the above findings, we hypothesized that FGF-2 may affect $S D F 1$ expression in pulp cells and modulate DPSC/MSC migration and odontoblast differentiation, and may thus have potential therapeutic applications. Surprisingly, our results indicated that treatment with FGF-2 for $48 \mathrm{~h}$ decreased $S D F 1$ expression to $<10 \%$ that of control pulp cells. FGF-2 transmits signals via receptor-type tyrosine kinases. Following the activation of a tyrosine kinase, various signaling pathways, such as mitogenactivated protein kinase (MAPK), protein kinase $\mathrm{C}$ (PKC) and phosphoinositol 3-kinase (PI3K) are triggered (32). In line with previous reports $(10,32)$, our data demonstrated that the FGF receptor antagonist, AZD4547, abolished the effects of FGF-2 in pulp cells. Since AZD4547 is an FGFR-specific tyrosine kinase inhibitor, the decreased mRNA expression of $S D F 1$ in the SDP11 cells treated with FGF-2 was thought to result from the modulation of FGF-2 via FGFR. However, SB203580 (a p38 inhibitor), PD98059 [an extracellular signal-regulated kinase (ERK) inhibitor], SP600125 [a c-Jun N-terminal kinase (JNK) inhibitor] and LY294002 (a PI3K inhibitor) did not inhibit the effects of FGF-2 (data not shown). Thus, the intracellular signaling pathways dependent on the mRNA expression of SDF1 in SDP11 cells and MSCs remain unclear, and further studies are required to examine these effects. The elucidation of the FGF-2-dependent signaling pathways in pulp cells may be important for identifying therapeutic applications for FGF-2 in the repair of dentin.

In conclusion, in the present study, we demonstrate that DPCs may be important for maintaining the homeostasis of DP tissue by controlling the migration of postnatal stem cells, e.g., MSCs and EPCs, to the required sites via SDF1-CXCR4 expression. Moreover, as SDF1 expression may be regulated by FGF-2 in DPCs, several other cytokines may be crucial for SDF1-CXCR4 expression and thus also for cell-based tissue regeneration. These findings may facilitate our understanding of the mechanisms of homeostasis in the DP via SDF1 expression.

\section{Acknowledgements}

The present study was supported in part by Grants-in-Aid for Scientific Research (C) (no. 25463182 to T.H.) and (B) (no. 26293435 to T.I.), Grants-in Aid for Exploratory Research (no. 25670869 to T.I.), and Grants-in-Aid for Young Scientists (B) (no. 26861790 to Y.A.) from the Ministry of Education, Culture, Sports, Science and Technology (MEXT); the Japan Society for the Promotion Science (JSPS); and the Futokukai Foundation (to Y.A., 2013).

\section{References}

1. Hasegawa T, Chosa N, Asakawa T, Yoshimura Y, Asakawa A, Ishisaki A and Tanaka M: Effect of fibroblast growth factor-2 on dental pulp cells derived from human deciduous teeth in vitro. Exp Ther Med 1: 477-480, 2010.

2. Orchardson R and Cadden SW: An update on the physiology of the dentine-pulp complex. Dent Update 28: 200-206, 208-209, 2001. 
3. Miura M, Gronthos S, Zhao M, Lu B, Fisher LW, Robey PG and Shi S: SHED: Sstem cells from human exfoliated deciduous teeth. Proc Natl Acad Sci USA 100: 5807-5812, 2003.

4. Arakaki M, Ishikawa M, Nakamura T, Iwamoto T, Yamada A, Fukumoto E, Saito M, Otsu K, Harada H, Yamada Y and Fukumoto S: Role of epithelial-stem cell interactions during dental cell differentiation. J Biol Chem 287: 10590-10601, 2012.

5. Fakhry M, Hamade E, Badran B, Buchet R and Magne D: Molecular mechanisms of mesenchymal stem cell differentiation towards osteoblasts. World J Stem Cells 5: 136-148, 2013.

6. Wang J, Loberg R and Taichman RS: The pivotal role of CXCL12 (SDF-1)/CXCR4 axis in bone metastasis. Cancer Metastasis Rev 25: 573-587, 2006.

7. Zernecke A, Schober A, Bot I, von Hundelshausen P, Liehn EA Möpps B, Mericskay M, Gierschik P, Biessen EA and Weber C: SDF-1 $\alpha / C X C R 4$ axis is instrumental in neointimal hyperplasia and recruitment of smooth muscle progenitor cells. Circ Res 96 784-791, 2005.

8. Kucia M, Ratajczak J, Reca R, Janowska-Wieczorek A and Ratajczak MZ: Tissue-specific muscle, neural and liver stem/progenitor cells reside in the bone marrow, respond to an SDF-1 gradient and are mobilized into peripheral blood during stress and tissue injury. Blood Cells Mol Dis 32: 52-57, 2004.

9. Ratajczak MZ, Kucia M, Reca R, Majka M, JanowskaWieczorek A and Ratajczak J: Stem cell plasticity revisited CXCR4-positive cells expressing mRNA for early muscle, liver and neural cells 'hide out' in the bone marrow. Leukemia 18 29-40, 2004.

10. Hasegawa T, Chosa N, Asakawa T, Yoshimura Y, Fujihara Y, Kitamura T, Tanaka M, Ishisaki A and Mitome M: Differential effects of TGF- $\beta 1$ and FGF- 2 on SDF- $1 \alpha$ expression in human periodontal ligament cells derived from deciduous teeth in vitro. Int J Mol Med 30: 35-40, 2012.

11. Mori T, Kiyono T, Imabayashi $\mathrm{H}$, Takeda $\mathrm{Y}$, Tsuchiya $\mathrm{K}$, Miyoshi S, Makino H, Matsumoto K, Saito H, Ogawa S, et al: Combination of hTERT and bmi-1, E6, or E7 induces prolongation of the life span of bone marrow stromal cells from an elderly donor without affecting their neurogenic potential. Mol Cell Biol 25: 5183-5195, 2005 .

12. Shimomura T, Yoshida Y, Sakabe T, Ishii K, Gonda K, Murai R, Takubo K, Tsuchiya H, Hoshikawa Y, Kurimasa A, et al: Hepatic differentiation of human bone marrow-derived UE7T-13 cells: Effects of cytokines and CCN family gene expression. Hepato Res 37: 1068-1079, 2007

13. Aomatsu E, Takahashi N, Sawada S, Okubo N, Hasegawa T, Taira M, Miura H, Ishisaki A and Chosa N: Novel SCRG1/BST1 axis regulates self-renewal, migration, and osteogenic differentiation potential in mesenchymal stem cells. Sci Rep 4: 3652, 2014.

14. Lv Z, Yang D, Li J, Hu M, Luo M, Zhan X, Song P, Liu C, Bai H, $\mathrm{Li}$ B, et al: Bone morphogenetic protein 9 overexpression reduces osteosarcoma cell migration and invasion. Mol Cells 36: 119-126, 2013.

15. Kimura K, Nakano T, Park YB, Tani M, Tsuda H, Beppu Y, Moriya $\mathrm{H}$ and Yokota J: Establishment of human osteosarcoma cell lines with high metastatic potential to lungs and their utilities for therapeutic studies on metastatic osteosarcoma. Clin Exp Metastasis 19: 477-485, 2002.

16. Hasegawa T, Chosa N, Asakawa T, Yoshimura Y, Ishisaki A and Tanaka M: Establishment of immortalized human periodontal ligament cells derived from deciduous teeth. Int J Mol Med 26: 701-705, 2010

17. Trubiani O, Isgro A, Zini N, Antonucci I, Aiuti F, Di Primio R, Nanci A, Caputi S and Paganelli R: Functional interleukin-7/interleukin-7Ralpha, and SDF-1alpha/CXCR4 are expressed by human periodontal ligament derived mesenchymal stem cells. J Cell Physiol 214: 706-713, 2008.

18. Vergote D, Butler GS, Ooms M, Cox JH, Silva C, Hollenberg MD, Jhamandas JH, Overall CM and Power C: Proteolytic processing of SDF-1alpha reveals a change in receptor specificity mediating HIV-associated neurodegeneration. Proc Natl Acad Sci USA 103: 19182-19187, 2006.
19. Nagasawa T, Kikutani $\mathrm{H}$ and Kishimoto $\mathrm{T}$ : Molecular cloning and structure of a pre-B-cell growth-stimulating factor. Proc Natl Acad Sci USA 91: 2305-2309, 1994.

20. Nagasawa T, Hirota S, Tachibana K, Takakura N, Nishikawa S, Kitamura Y, Yoshida N, Kikutani H and Kishimoto T: Defects of B-cell lymphopoiesis and bone-marrow myelopoiesis in mice lacking the CXC chemokine PBSF/SDF-1. Nature 382: 635-638, 1996.

21. Tachibana K, Hirota S, Iizasa H, Yoshida H, Kawabata K, Kataoka Y, Kitamura Y, Matsushima K, Yoshida N, Nishikawa S, et al: The chemokine receptor CXCR4 is essential for vascularization of the gastrointestinal tract. Nature 393: 591-594, 1998

22. Sugiyama T, Kohara H, Noda M and Nagasawa T: Maintenance of the hematopoietic stem cell pool by CXCL12-CXCR4 chemokine signaling in bone marrow stromal cell niches. Immunity 25 : 977-988, 2006.

23. Yano T, Liu Z, Donovan J, Thomas MK and Habener JF: Stromal cell derived factor-1 (SDF-1)/CXCL12 attenuates diabetes in mice and promotes pancreatic beta-cell survival by activation of the prosurvival kinase Akt. Diabetes 56: 2946-2957, 2007.

24. Tsuchiya A, Imai M, Kamimura H, Takamura M, Yamagiwa $S$, Sugiyama T, Nomoto M, Heike T, Nagasawa T, Nakahata T, et al: Increased susceptibility to severe chronic liver damage in CXCR4 conditional knock-out mice. Dig Dis Sci 57: 2892-2900, 2012.

25. Asano Y, Iimuro Y, Son G, Hirano T and Fujimoto J: Hepatocyte growth factor promotes remodeling of murine liver fibrosis, accelerating recruitment of bone marrow-derived cells into the liver. Hepatol Res 37: 1080-1094, 2007.

26. Saxena A, Fish JE, White MD, Yu S, Smyth JW, Shaw RM, DiMaio JM and Srivastava D: Stromal cell-derived factor-1alpha is cardioprotective after myocardial infarction. Circulation 117: 2224-2231, 2008

27. Farges JC, Keller JF, Carrouel F, Durand SH, Romeas A, Bleicher F, Lebecque $S$ and Staquet MJ: Odontoblasts in the dental pulp immune response. J Exp Zoolog B Mol Dev Evol 312B: 425-436, 2009.

28. Zhou J, Shi S, Shi Y, Xie H, Chen L, He Y, Guo W, Wen L and Jin Y: Role of bone marrow-derived progenitor cells in the maintenance and regeneration of dental mesenchymal tissues. J Cell Physiol 226: 2081-2090, 2011.

29. Kimura Y, Komaki M, Iwasaki K, Sata M, Izumi Y and Morita I: Recruitment of bone marrow-derived cells to periodontal tissue defects. Front Cell Dev Biol 2: 19, 2014.

30. Luster AD: Chemokines - chemotactic cytokines that mediate inflammation. N Engl J Med 338: 436-445, 1998.

31. Ratajczak MZ, Zuba-Surma E, Kucia M, Reca R, Wojakowski W and Ratajczak J: The pleiotropic effects of the SDF-1-CXCR4 axis in organogenesis, regeneration and tumorigenesis. Leukemia 20: 1915-1924, 2006

32. Murakami S: Periodontal tissue regeneration by signaling molecule(s): What role does basic fibroblast growth factor (FGF-2) have in periodontal therapy? Periodontol 56: 188-208, 2011.

33. Fujii S, Maeda H, Tomokiyo A, Monnouchi S, Hori K, Wada N and Akamine A: Effects of TGF- $\beta 1$ on the proliferation and differentiation of human periodontal ligament cells and a human periodontal ligament stem/progenitor cell line. Cell Tissue Res 342: 233-242, 2010.

34. Kim YS, Min KS, Jeong DH, Jang JH, Kim HW and Kim EC: Effects of fibroblast growth factor- 2 on the expression and regulation of chemokines in human dental pulp cells. J Endod 36: 1824-1830, 2010.

35. Goldberg M and Smith AJ: Cells and extracellular matrices of dentin and pulp: A biological basis for repair and tissue engineering. Crit Rev Oral Biol Med 15: 13-27, 2004.

36. Schmidt A, Ladage D, Schinköthe T, Klausmann U, Ulrichs C, Klinz FJ, Brixius K, Arnhold S, Desai B, Mehlhorn U, et al: Basic fibroblast growth factor controls migration in human mesenchymal stem cells. Stem Cells 24: 1750-1758, 2006. 